\title{
Knockdown of microtubule actin crosslinking factor 1 inhibits cell proliferation in MC3T3-E1 osteoblastic cells
}

\author{
Lifang Hu, Peihong Su, Runzhi Li, Kun Yan, Zhihao Chen, Peng Shang \& Airong Qian* \\ Key Laboratory for Space Bioscience and Biotechnology, Institute of Special Environmental Biophysics, School of Life Sciences, \\ Northwestern Polytechnical University, Xi'an, Shaanxi 710072, P. R. China
}

\begin{abstract}
Microtubule actin crosslinking factor 1 (MACF1), a widely expressed cytoskeletal linker, plays important roles in various cells by regulating cytoskeleton dynamics. However, its role in osteoblastic cells is not well understood. Based on our previous findings that the association of MACF1 with F-actin and microtubules in osteoblast-like cells was altered under magnetic force conditions, here, by adopting a stable MACF1knockdown MC3T3-E1 osteoblastic cell line, we found that MACF1 knockdown induced large cells with a binuclear/multinuclear structure. Further, immunofluorescence staining showed disorganization of F-actin and microtubules in MACF1-knockdown cells. Cell counting revealed significant decrease of cell proliferation and cell cycle analysis showed an $\mathrm{S}$ phase cell cycle arrest in MACF1-knockdown cells. Moreover and interestingly, MACF1 knockdown showed a potential effect on cellular MTT reduction activity and mitochondrial content, suggesting an impact on cellular metabolic activity. These results together indicate an important role of MACF1 in regulating osteoblastic cell morphology and function. [BMB Reports 2015; 48(10): 583-588]
\end{abstract}

\section{INTRODUCTION}

Microtubule actin crosslinking factor 1 (MACF1), also known as actin crosslinking family 7 (ACF7), is a member of the plakin family of large structural proteins that link cytoskeletal elements (1-3). MACF1 is a $\sim 600 \mathrm{kDa}$ cytoskeletal linker that can bind to both F-actin and microtubules (4-6). MACF1 is widely expressed in tissues (7) and is well known for its critical role in controlling the actin and microtubule cytoskeletal dynamics $(2,8,9)$. MACF1 performs key roles in numerous cell

${ }^{*}$ Corresponding author. Tel: +86-29-88491840; Fax: +86-2988491840; E-mail: qianair@nwpu.edu.cn

http://dx.doi.org/10.5483/BMBRep.2015.48.10.098

Received 21 May 2015, Revised 16 June 2015, Accepted 6 August 2015

Keywords: Cell morphology, Cell proliferation, Cytoskeleton, MACF1, Osteoblastic cell types, including epidermal cells, neuronal cells, hair cells and others $(2,8,10,11)$.

We have previously found MACF1 to be widely expressed in MC3T3-E1 osteoblastic cells and to partially co-localize with F-actin and microtubules (12). During the osteoblast response to environmental stimuli, the distribution of MACF1 and its co-localization with F-actin and microtubules were altered. Thus, we hypothesized that MACF1 might be crucial in regulating osteoblast function, based on the role of MACF1 in non-osteoblastic cells and our previous findings. However, the function of MACF1 in osteoblastic cells is not well known.

In this study, to advance our understanding of MACF1 in osteoblastic cells, we adopted a stable MACF1-knockdown cell line established with lentivirus-mediated shRNA technology in MC3T3-E1 osteoblastic cells and determined the effects of MACF1 knockdown on cell morphology, cytoskeleton distribution and cell proliferation. We demonstrated that MACF1 knockdown altered cell morphology, increased binuclear/multinuclear cell percentage, disrupted the normal distribution of F-actin and microtubules, and inhibited cell proliferation by inducing S phase cell cycle arrest in MC3T3-E1 cells. Moreover, MACF1 knockdown showed a potential effect on cellular metabolic activity. These results together, for the first time, indicate a direct role of MACF1 in regulating osteoblastic cell morphology and function and provide new insights into both MACF1 and osteoblasts.

\section{RESULTS}

MACF1 knockdown altered the morphology of MC3T3-E1 cells and increased the binuclear/multinuclear cell percentage After determining that the MACF1 expression was significantly reduced in the stable MACF1-knockdown (KD) cells at both mRNA level $(60-80 \%$, Fig. $1 \mathrm{~A})$ and protein level $(\sim 100 \%$, Fig. 1B) compared with the parental MC3T3-E1 cells and the scrambled controls, the cell morphology was examined by either phase contrast microscopy (Fig. 1C) or hematoxylin-eosin (HE) staining (Fig. 1D). Both the parental MC3T3-E1 cells and the scrambled control cells showed fusiform and polygonal morphology, typical characteristics of osteoblasts, while MACF1 knockdown induced larger cells, often presenting as binuclear/multinuclear structure (Fig. 1C, D). Further calculation 
with the DAPI stained nucleus (Fig. S1) showed a significant increase of the binuclear/multinuclear cell percentage in the MACF1-knockdown cells (Fig. 1E). These findings suggested that MACF1 knockdown altered cell morphology and might cause cytokinesis defect.

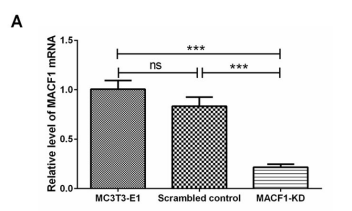

B
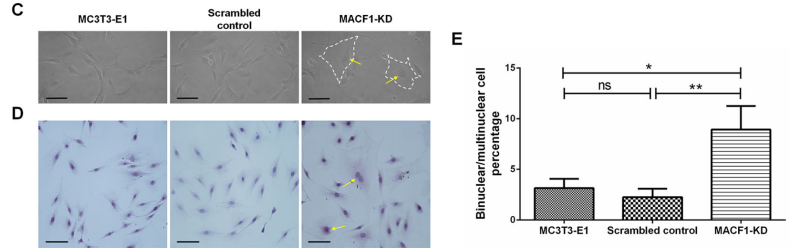

Fig. 1. Knockdown efficiency of lentivirus-mediated shRNA on MACF1 expression in stable, transfected MC3T3-E1 osteoblastic cells and the effect of MACF1 knockdown on cell morphology. (A) Real time PCR analysis and (B) western blot detection showed significant reduction of MACF1 expression in the MACF1-knockdown (KD) cells at both mRNA level and protein level, compared with the parental MC3T3-E1 cells and the scrambled controls (***P $<0.001)$. (C, D) Cell morphology of the parental MC3T3-E1 cells, scrambled controls and MACF1-knockdown (KD) cells was observed using either inverted phase contrast microscopy (C) or HE staining (D) after a $24 \mathrm{~h}$ culture. MACF1 knockdown induced large binuclear/multinuclear cells. The white dashed line indicates the large cell border and the yellow arrows indicate the binuclear/multinuclear structures. Scale bar $=100$ $\mu \mathrm{m}$. (E) Quantification of the percentage of binuclear/multinuclear cells $\left({ }^{*} \mathrm{P}<0.05, * * \mathrm{P}<0.01\right)$. The data represent the mean $\pm \mathrm{SD}$.

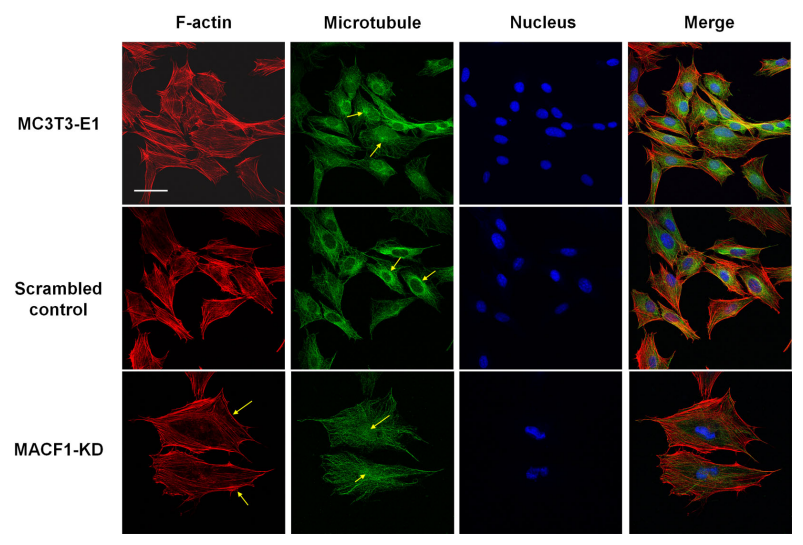

Fig. 2. MACF1 knockdown induced the redistribution of F-actin and microtubules. Cells were stained with phalloidin, tubulin, and DAPI. MACF1 knockdown disrupted the normal distribution of Factin (red) and microtubules (green). Yellow arrows indicate the localization of the F-actin, the distribution and MTOC for the microtubules. Scale bar $=50 \mu \mathrm{m}$.

\section{MACF1 knockdown induced the redistribution of the cytoskeleton}

MACF1 plays key role in regulating the dynamics of F-actin and microtubules $(2,8)$, which are essential for maintaining cell shape and involved in cytokinesis. We further determined the effects of MACF1 knockdown on the F-actin and microtubules by immunofluorescence staining. As shown in Fig. 2, in both the parental MC3T3-E1 cells and the scrambled control cells, F-actin (red, rhodamine) was distributed at the cell periphery or passed through the cytoplasm as rope-like structures, microtubules (green, FITC) radiated from a microtubuleorganizing center (MTOC) to the cell plasma membrane, presenting as tortuous filaments, and the nuclei (blue, DAPI) were regularly shaped. While, in the MACF1-knockdown cells, the F-actin filaments became thinner and were mainly localized at the cell periphery, and the microtubules were reorganized, resulting in longer, straighter filaments with loss of the MTOC in association with compact $\alpha$-tubulin accumulation around and in the nucleus (Fig. 2).

\section{MACF1 knockdown inhibited cell proliferation and induced} $S$ phase cell cycle arrest in MC3T3-E1 cells

Based on the above results, we wondered whether the cell proliferation was affected by MACF1 knockdown. The cell counting detection showed that MACF1 knockdown significantly decreased the cell number after $24 \mathrm{~h}, 48 \mathrm{~h}$, and $72 \mathrm{~h}$ of cell culture (Fig. 3A), without causing cell death (Fig. S2), suggesting an inhibitory effect of MACF1 knockdown on cell proliferation. The cell cycle analysis (Fig. 3B) showed a significant

A

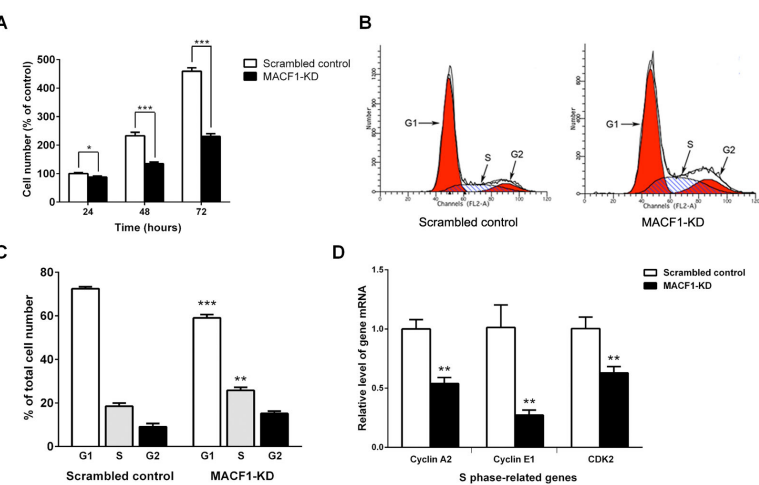

Fig. 3. MACF1 knockdown inhibited cell proliferation and induced $\mathrm{S}$ phase cell cycle arrest in MC3T3-E1 cells. (A) After $24 \mathrm{~h}, 48 \mathrm{~h}$, and $72 \mathrm{~h}$ of cell culture, the scrambled control cells and MACF1KD cells were harvested and automatically counted using Vi-Cell XR cell viability analyzer. The cell proliferation was significantly inhibited by MACF1 knockdown ( $* \mathrm{P}<0.05$, ***P $<0.001)$. (B, C) Cell cycle analysis by flow cytometry (B) showed that MACF1 knockdown dramatically increased the $S$ phase cell percentage $(C)$, MACF1- KD versus scrambled control, **P $<0.01, * * * P<0.001$. (D) Real time PCR analysis showed significant down-regulation of $\mathrm{S}$ phase-related genes expression, MACF1-KD versus scrambled control, $* * P<0.01$. The data represent the mean \pm SD. 
increase of the S phase cell percentage in MACF1-knockdown cells $(139.4 \%$ as scrambled control, Fig. 3C). Further examination also showed remarkable reduction of the expression of critical S phase-related genes, including cyclin A2, cyclin E1, and cyclin-dependent kinase 2 (CDK2), in the MACF1-knockdown cells (Fig. 3D). These results suggested that the inhibition of cell proliferation by MACF1 knockdown might occur through the induction of an $S$ phase cell cycle arrest.

\section{The effect of MACF1 knockdown on cellular MTT reduction activity and mitochondrial content}

As cell proliferation and cell cycling process are related with cell metabolic status, the effects of MACF1 knockdown on cellular metabolic activity was further examined using the MTT (3-(4,5-dimethylthiazol-2-yl)-2,5-diphenyltetrazolium bromide) assay, which reflects cellular metabolic activity by measuring the conversion of MTT into purple-colored MTT formazan crystals by mitochondrial dehydrogenases (13). The results showed that MACF1 knockdown did not cause significant changes in the absorbance after $24 \mathrm{~h}, 48 \mathrm{~h}$ and $72 \mathrm{~h}$ of cell culture (Fig. 4A), indicating that the cellular MTT reduction activity was not affected. This finding is interesting for its disagreement with the cell proliferation results (Fig. 3A). Because the MTT assay is frequently used to detect cell proliferation based on the theory that the MTT reduction activity correlates with the number of living cells $(13,14)$. However, as shown in Fig. 3A, the cell proliferation was significantly inhibited by MACF1 knockdown.

To determine a possible explanation for this result, the absorbance of the same number of cells was measured. As expected, the data showed that the absorbance of MACF1-knock- down cells was substantially higher than that of the same number of the scrambled controls $(\mathrm{P}<0.05$, Fig. 4B). Previous reports have demonstrated the relationship between cell size, cell cycle and mitochondria activity (15-17). Therefore, based on our findings, we hypothesized that the MACF1 knockdown-induced increase of absorbance may be due to the increased large binuclear/multinuclear cells. To assess this, we examined the active mitochondrial content using MitoTracker Green staining. Compared with the scrambled control, more mitochondrial content were observed in the large binuclear/ multinuclear cells (Fig. 4C). Thus, our findings indicated that MACF1 knockdown increased the population of large cells, and consequently mitochondrial content and metabolic activity.

\section{DISCUSSION}

MACF1 plays key role in regulating the dynamics of F-actin and microtubule cytoskeleton, and has been demonstrated roles in numerous cell types $(2,8-10,18)$. Here, we demonstrate for the first time the role of MACF1 in osteoblastic cells using MACF1-knockdown osteoblastic cells, based on our previous findings (12). The results showed that MACF1 knockdown altered cell morphology, disrupted the distribution of F-actin and microtubules, inhibited cell proliferation, and induced $\mathrm{S}$ phase cell cycle arrest.

Cell morphology, the basis of cell function, was first examined. The parental MC3T3-E1 cells and the scrambled control cells exhibited fusiform and polygonal morphology, while there were significant increased population of large, binuclear/ multinuclear cells (Fig. 1C-E), suggesting that there might be alterations in the cytoskeleton and cytokinesis.
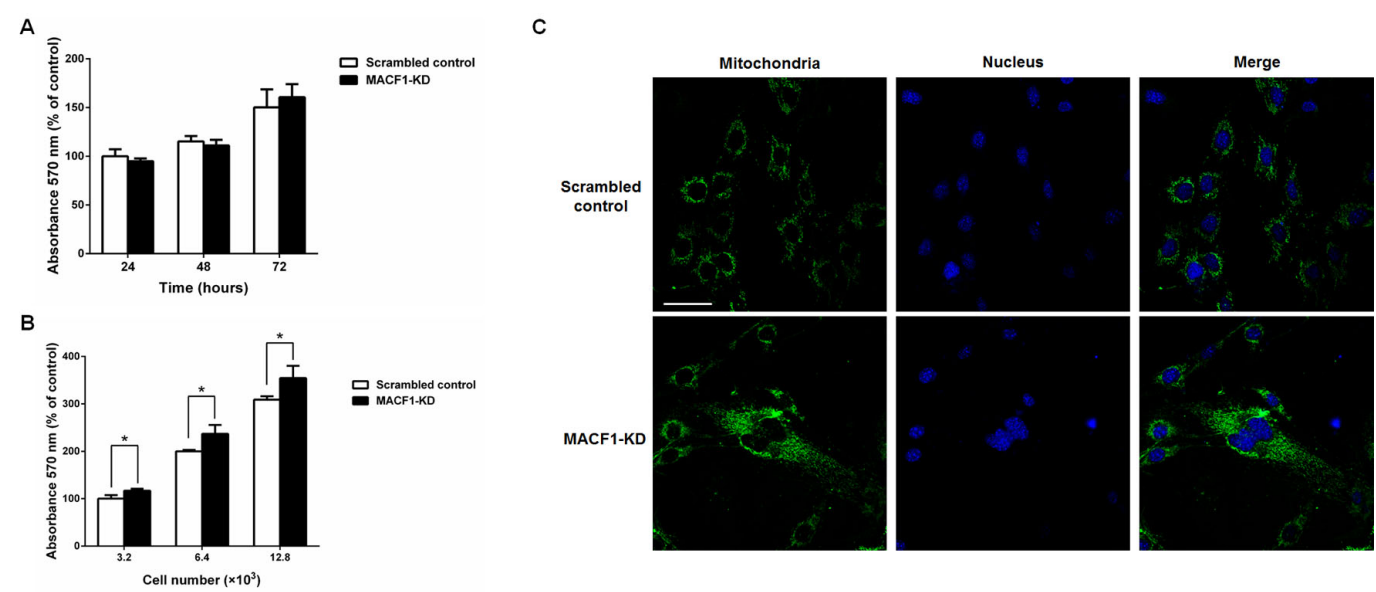

Fig. 4. The effect of MACF1 knockdown on cellular MTT reduction activity and mitochondrial content. (A) After $24 \mathrm{~h}$, $48 \mathrm{~h}$, and $72 \mathrm{~h}$ of cell culture, the MTT assay was conducted, showing no significant difference in the absorbance between the scrambled control and MACF1-KD cells. (B) Detection of the absorbance of the same cell number in the scrambled control or the MACF1-KD group showed increased cellular MTT reduction activity by MACF1 knockdown. MACF1-KD versus scrambled control, ${ }^{*} \mathrm{P}<0.05$. (C) Cells were stained with MitoTracker green and DAPI, more mitochondrial content (green) was observed in the large binuclear/multinuclear cells. Scale bar $=50$ m. 
Further investigations confirmed our hypothesis. Striking Factin and microtubule changes occurred in the MACF1-knockdown cells (Fig. 2). F-actin became thinner and was mainly localized at the cell periphery, and microtubule filaments became straight and longer associated with the disappearance of MTOC. This finding was consistent with the results of Kodama et al. (2). They also found that ACF7 (MACF1) deficiency resulted in less stable, longer microtubules associated with defective polarization of the MTOC in endodermal cells. These results indicate the importance of MACF1 in regulating cytoskeletal dynamics.

Disruption of the normal dynamics of F-actin or microtubules causes cytokinesis defects that result in binuclear/multinuclear cells and cell cycle arrest, which affects cell proliferation (19-21). Thus we wondered whether the cell proliferation would be changed by the MACF1 knockdown. As expected, MACF1 knockdown dramatically inhibited the cell proliferation (Fig. 3A). In addition, further cell cycle analysis showed that MACF1 knockdown induced an S phase cell cycle arrest in association with the down-regulation of $\mathrm{S}$ phaserelated genes (Fig. 3B-D). These findings suggest that the inhibitory effect of MACF1 knockdown on cell proliferation may through the induction of $\mathrm{S}$ phase arrest. Although we found that MACF1 knockdown dramatically inhibited cell proliferation and caused cytokinesis defective characteristic in MC3T3-E1 osteoblastic cells, Wu et al. reported that ACF7 absence did not cause significant decrease of cell proliferation or mitosis defects in either epidermal or endodermal cells $(2,8,9)$. This difference may be due to the different cell types. Menon et al. have reported a cell-type-specific requirement of the core septin SEPT7, a cytoskeletal protein, for cytokinesis (22). Besides, as cytokinesis is a complex process that involves many proteins (23), we wonder that there may be additional as-yet unidentified osteoblastic cell-specific proteins that interact with MACF1 in regulating cytokinesis. Further studies need to be carried out.

Another interesting finding was that MACF1 knockdown increased the cellular MTT reduction activity (Fig. 4B) as this was in contrast with the cell number result. Previous studies have reported the discrepancies between MTT assay and cell counting, and revealed that the cellular MTT reduction activity was related with mitochondrial content and activity rather than cell number (16). In addition, a strong coupling between cell size and mitochondrial content has been demonstrated (17). Moreover, there is correlation between cell cycle and mitochondrial activity, showing that the cell size increases when cell entering $S$ phase, together with increased mitochondrial activity (15). We also found a greater mitochondrial content in the large binuclear/multinuclear cells in MACF1-knockdown group (Fig. 4C). Thus, our findings suggest that the MACF1 knockdowninduced the increase of cellular MTT reduction activity may be due to the increased number of large binuclear/multinuclear cells, which show more active mitochondrial content.

In conclusion, present study demonstrates for the first time the role of MACF1 in osteoblastic cells. Our results suggest an essential and positive role of MACF1 in maintaining cell morphology, cytoskeleton organization and cell proliferation. Furthermore, this work demonstrates that the inhibitory effect of MACF1 knockdown on cell proliferation may be due to a cytokinesis defect and an $\mathrm{S}$ phase cell cycle arrest. Moreover, present studies indicates a potential effect of MACF1 knockdown on cellular metabolic ability by increasing large binuclear/multinuclear cells, and consequently the mitochondrial content. Further studies including the in vivo experiments will be carried out in future.

\section{MATERIALS AND METHODS}

\section{Cell culture and construction of stable MACF1-knockdown cell line}

The murine MC3T3-E1 osteoblastic cells were provided by Dr. Hong Zhou of the University of Sydney. MC3T3-E1 cells were cultured in $\alpha$-MEM medium (Life Technologies, USA) supplemented with $10 \%$ fetal bovine serum (FBS) (Life Technologies, USA), $100 \mu \mathrm{g} / \mathrm{ml}$ streptomycin and 100 units $/ \mathrm{ml}$ penicillin, in a humidified, $37^{\circ} \mathrm{C}, 5 \% \mathrm{CO}_{2}$ incubator. For the construction of the stable MACF1-knockdown osteoblastic cell line, shRNA specifically targeting murine MACF1 (NM 001199136.1) and one scrambled shRNA were designed and synthesized by Genepharma Co. Ltd (Shanghai, China). MC3T3-E1 cells were transfected with either MACF1-shRNA lentivirus vector or scrambled shRNA vector. Finally, the stably transfected cell lines were selected under the same selection condition with puromycin, and the knockdown efficiency was determined using both real time RT-PCR and western blot.

\section{Real time RT-PCR}

Real time RT-PCR was performed as previously described (12). Briefly, total RNA was extracted from cells using TRIzol reagent (Invitrogen, USA) and reverse transcribed into complementary DNA (cDNA). Then, real time PCR detection of gene expression was performed with specific primers and SYBR Green using $\beta$-actin or GAPDH as an internal control. The thermal cycling conditions included initial denaturation step at $95^{\circ} \mathrm{C}$ for $30 \mathrm{~s}, 40$ cycles at $95^{\circ} \mathrm{C}$ for $10 \mathrm{~s}, 60^{\circ} \mathrm{C}$ for $20 \mathrm{~s}, 72^{\circ} \mathrm{C}$ for $5 \mathrm{~s}$. The relative expression was calculated via $2^{-\Delta \Delta \mathrm{Ct}}$ method (24). The gene specific primers are: MACF1, sense: (5'-GAAAACATTCACCAAGTGGGTCAAC-3') and antisense (5'-TGTCCATCCCGAAGGTCTTCATAG-3'); cyclin A2, sense (5'-AGTACСTGCСТTCACTCATTGCTG-3') and antisense (5'-TCTGGTGAAGGTCCACAAGACAAG-3'); cyclin E1, sense: (5'-GCTTCGGGTCTGAGTTCCAA-3') and antisense (5'-GGATGAAAGAGCAGGGGTCC-3'); CDK2, sense: (5'-TGTGCCTCCCCTGGATGAAG-3') and antisense (5'-CATCCTGGAAGAAAGGGTGA-3'); $\beta$-actin, sense: (5'-AGTGTGACGTTGACATCCGTA-3') and antisense (5'-GCCAGAGCAGTAATCTCCTTCT-3'); GAPDH, sense (5'-AGTGTGACGTTGACATCCGTA-3') and antisense (5'GCCAGAGCAGTAATCTCCTTCT-3'). 


\begin{abstract}
Western blot
Protein extraction was performed using cell lysis buffer (50 $\mathrm{mM}$ Tris-base, $1 \mathrm{mM}$ EDTA, $150 \mathrm{mM} \mathrm{NaCl}, 0.1 \%$ SDS, $1 \%$ Triton X-100, and $1 \mathrm{mM}$ PMSF) on ice. Equal amount of proteins were subjected to 6\% SDS-PAGE and transblotted to PVDF membrane. After incubation with the blocking buffer (5\% nonfat milk), the membrane was subjected for overnight incubation at $4^{\circ} \mathrm{C}$ with primary antibody against MACF1 (Abcam, USA), or GAPDH (Sigma-Aldrich, USA). The horseradish peroxidase (HRP) conjugated secondary antibody was further used. Protein bands were visualized by chemiluminescence using an ECL kit (Pierce, USA) and exposed to X-ray film.
\end{abstract}

\section{Cell morphology observation}

Cell morphology was observed using an inverted phase contrast microscope (Olympus, Japan) and images were captured. In addition, HE staining was applied to determine the changes in cell morphology using the Hematoxylin and Eosin Staining Kit (Beyotime Institute of Biotechnology, China) as previously described (25). Cells $\left(1 \times 10^{4} / \mathrm{cm}^{2}\right)$ were seeded on coverslips and cultured for $24 \mathrm{~h}$. Then, cells were fixed with $95 \%$ ethanol, stained with $\mathrm{HE}$, and observed under a microscope (Nikon $80 \mathrm{i}$, Japan) with images being captured.

\section{Immunofluorescence staining}

Cells $\left(1 \times 10^{4} / \mathrm{cm}^{2}\right)$ were seeded on coverslips. After a $24 \mathrm{~h}$ culture, immunofluorescence staining of F-actin, $\alpha$-tubulin and the nucleus was conducted. After 15-min fixation with $4 \%$ paraformaldehyde and permeation with $0.5 \%$ Triton X-100 (TBS), the cells were stained with mouse rhodamine phalloidin (1:40, Invitrogen, USA) and anti- $\alpha$-tubulin (1:20, Calbiochem, USA) overnight at $4^{\circ} \mathrm{C}$. Then, the cells were incubated with goat anti-mouse FITC-IgG secondary antibody (1:100, CoWin Biotech Co, China) for 1 h. 4',6-diamidino-2-phenylindole (DAPI, 1 $\mu \mathrm{g} / \mathrm{ml}$ ) was used to counterstain the nucleus. The cells were enveloped with Fluoromount-G (SouthernBiotech, USA) and visualized using a laser confocal scanning microscope (Leica TCS SP5, Germany) or a fluorescence microscope (Nikon 80i, Japan). Images were captured, and the percentage of binuclear/ multinuclear cells was determined from more than 200 cells and statistically analyzed using the GraphPad Prism software.

\section{Cell proliferation assay}

The cell proliferation and viability was detected using Vi-Cell XR cell viability analyzer (Beckman Coulter, USA) as previously described $(26,27)$. This technique detects the cell number and cell viability by applying trypan blue dye-exclusion staining combined with image-based data analysis. Cells were plated into 6-well plates in triplicate at a density of $1 \times 10^{4} / \mathrm{cm}^{2}$ and cultured for $24 \mathrm{~h}, 48 \mathrm{~h}$ and $72 \mathrm{~h}$. Then, the cells were harvested by digestion with trypsin/EDTA and resuspended in $1 \mathrm{ml}$ medium for automatic cell counting with Vi-Cell analyzer. The cell number and percentage of living cells were determined. The percentage of living cells was pre- sented as number of viable cells/total number of cells $\times 100 \%$.

\section{Cell cycle analysis}

Cells $\left(1 \times 10^{4} / \mathrm{cm}^{2}\right)$ were incubated for $48 \mathrm{~h}$ and harvested. The cells were fixed in $70 \%$ ice-cold ethanol overnight at $4^{\circ} \mathrm{C}$. After washing with PBS, cells were stained with PI solution (1 $\mathrm{mg} / \mathrm{ml}$ sodium citrate, $0.3 \%$ Triton $\mathrm{X}-100,0.1 \mathrm{mg} / \mathrm{ml} \mathrm{Pl}, 0.02$ $\mathrm{mg} / \mathrm{ml}$ RNase A) in the dark at $4^{\circ} \mathrm{C}$ for $30 \mathrm{~min}$. The cell cycle was determined by flow cytometer (FACSCalibur, BD Biosciences, USA) and the percentage of cells in the $G_{1}, S$ and $G_{2}$ phases was calculated using the ModFit software.

\section{MTT assay}

MTT assay measures the conversion of MTT into purple-colored MTT formazan by mitochondrial enzymes (13) and was performed as previously described (28). Cells were seeded into 96-well plates at a density of $1 \times 10^{4} / \mathrm{cm}^{2}$ in triplicate. After $24 \mathrm{~h}$, $48 \mathrm{~h}$ and $72 \mathrm{~h}$ of culture, $20 \mu \mathrm{l}$ of MTT $(5 \mathrm{mg} / \mathrm{ml})$ solution was added to 96-well plates and the cells were incubated for $4 \mathrm{~h}$ at $37^{\circ} \mathrm{C}$, followed by removal of the culture medium and the addition of $150 \mu$ of dimethyl sulfoxide (DMSO). After a $10 \mathrm{~min}$ vibration, the absorbance was measured at $570 \mathrm{~nm}$ on a multifunctional plate reader (Bio-Tek, USA) with the medium culture subtracted as the baseline. For further investigation, the MTT formazan absorbance of the same number of scrambled control and MACF1-knockdown cells $\left(3.2 \times 10^{3} /\right.$ well, $6.4 \times$ $10^{3} /$ well, or $12.8 \times 10^{3} /$ well) was determined. Briefly, cells at three different densities including $1 \times 10^{4} / \mathrm{cm}^{2}, 2 \times 10^{4} / \mathrm{cm}^{2}$, and $4 \times 10^{4} / \mathrm{cm}^{2}$, were seeded in 96 -well plates in triplicate and the absorbance was measured after a 12-h culture.

\section{Mitochondria detection}

Live cells were incubated with $150 \mathrm{nM}$ MitoTracker Green FM (Life Technologies, USA) for $45 \mathrm{~min}$ and the nucleus were counterstained with DAPI for $30 \mathrm{~min}$ at $37^{\circ} \mathrm{C}$ in $5 \% \mathrm{CO}_{2}$. Then, cells were imaged with a laser confocal scanning microscope using $488 \mathrm{~nm}$ excitation for MitoTracker Green and 405 nm excitation for DAPI.

\section{Statistical analysis}

All experiments were independently repeated at least three times with cells established in triplicate for each single assay, and the data were reported as the mean value \pm standard deviation (SD). Statistical analyses of the data were performed using the GraphPad Prism software, and a student $t$-test was used. $P$ values $<0.05$ were considered significant and indicated in the figures.

\section{ACKNOWLEDGEMENTS}

The authors would like to thank Dr. Hong Zhou (The University of Sydney, Australia) for her generous gift of the MC3T3-E1 cell line. This work was supported by grants from the National Natural Science Foundation of China (grant number 31400725, 
31570940), the Fundamental Research Funds for the Central Universities (3102014JKY15007), and the Project Funded by China Postdoctoral Science Foundation (grant number 2014M562450).

\section{REFERENCES}

1. Fuchs E and Karakesisoglou I (2001) Bridging cytoskeletal intersections. Genes Dev 15, 1-14

2. Kodama A, Karakesisoglou I, Wong E, Vaezi A and Fuchs E (2003) ACF7: an essential integrator of microtubule dynamics. Cell 115, 343-354

3. Leung CL, Green KJ and Liem RK (2002) Plakins: a family of versatile cytolinker proteins. Trends Cell Biol 12, 37-45

4. Leung $C L$, Sun D, Zheng $M$, Knowles DR and Liem RK (1999) Microtubule actin cross-linking factor (MACF): a hybrid of dystonin and dystrophin that can interact with the actin and microtubule cytoskeletons. J Cell Biol 147, $1275-1286$

5. Sun D, Leung CL and Liem RK (2001) Characterization of the microtubule binding domain of microtubule actin crosslinking factor (MACF): identification of a novel group of microtubule associated proteins. J Cell Sci 114, 161-172

6. Karakesisoglou I, Yang Y and Fuchs E (2000) An epidermal plakin that integrates actin and microtubule networks at cellular junctions. J Cell Biol 149, 195-208

7. Bernier G, Pool M, Kilcup M, Alfoldi J, De Repentigny $Y$ and Kothary R (2000) Acf7 (MACF) is an actin and microtubule linker protein whose expression predominates in neural, muscle, and lung development. Dev Dyn 219, 216-225

8. Wu X, Kodama A and Fuchs E (2008) ACF7 regulates cytoskeletal-focal adhesion dynamics and migration and has ATPase activity. Cell 135, 137-148

9. Wu X, Shen QT, Oristian DS et al (2011) Skin stem cells orchestrate directional migration by regulating microtubule-ACF7 connections through GSK3beta. Cell 144, 341-352

10. Sanchez-Soriano N, Travis M, Dajas-Bailador F, GoncalvesPimentel C, Whitmarsh AJ and Prokop A (2009) Mouse ACF7 and drosophila short stop modulate filopodia formation and microtubule organisation during neuronal growth. J Cell Sci 122, 2534-2542

11. Antonellis PJ, Pollock LM, Chou SW et al (2014) ACF7 is a hair-bundle antecedent, positioned to integrate cuticular plate actin and somatic tubulin. J Neurosci 34, 305-312

12. Qian AR, Hu LF, Gao X et al (2009) Large gradient high magnetic field affects the association of MACF1 with actin and microtubule cytoskeleton. Bioelectromagnetics 30, 545-555

13. Mosmann T (1983) Rapid colorimetric assay for cellular growth and survival: application to proliferation and cytotoxicity assays. J Immunol Methods 65, 55-63
14. Suzuki R, Matsuno S, Sakagami H, Okada Y and Shirataki Y (2014) Search of new cytotoxic crude materials against human oral squamous cell carcinoma using $1 \mathrm{H}$ NMRbased metabolomics. Anticancer Res 34, 4117-4120

15. Al-Rubeai M, Chalder S, Bird R and Emery AN (1991) Cell cycle, cell size and mitochondrial activity of hybridoma cells during batch cultivation. Cytotechnology 7, 179-186

16. Pagliacci MC, Spinozzi F, Migliorati G et al (1993) Genistein inhibits tumour cell growth in vitro but enhances mitochondrial reduction of tetrazolium salts: a further pitfall in the use of the MTT assay for evaluating cell growth and survival. Eur J Cancer 29A, 1573-1577

17. Kitami T, Logan DJ, Negri J et al (2012) A chemical screen probing the relationship between mitochondrial content and cell size. PLoS One 7, e33755

18. Ka M, Jung EM, Mueller U and Kim WY (2014) MACF1 regulates the migration of pyramidal neurons via microtubule dynamics and GSK-3 signaling. Dev Biol 395, 4-18

19. Moulding DA, Blundell MP, Spiller DG et al (2007) Unregulated actin polymerization by WASp causes defects of mitosis and cytokinesis in X-linked neutropenia. J Exp Med 204, 2213-2224

20. Hossain MM, Hwang DY, Huang QQ, Sasaki Y and Jin JP (2003) Developmentally regulated expression of calponin isoforms and the effect of h2-calponin on cell proliferation. Am J Physiol Cell Physiol 284, C156-167

21. Zhu J, Beattie EC, Yang Y, Wang HJ, Seo JY and Yang LX (2005) Centrosome impairments and consequent cytokinesis defects are possible mechanisms of taxane drugs. Anticancer Res 25, 1919-1925

22. Menon MB and Gaestel M (2015) Sep(t)arate or not - how some cells take septin-independent routes through cytokinesis. J Cell Sci 128, 1877-1886

23. D'Avino PP, Giansanti MG and Petronczki M (2015) Cytokinesis in animal cells. Cold Spring Harb Perspect Biol 7, a015834

24. Livak KJ and Schmittgen TD (2001) Analysis of relative gene expression data using real-time quantitative PCR and the $2^{-\Delta \Delta C T}$ Method. Methods 25, 402-408

25. Qian A, Zhang W, Weng Y et al (2008) Gravitational environment produced by a superconducting magnet affects osteoblast morphology and functions. Acta Astronautica 63, 929-946

26. Mundra JJ, Terskiy A and Howells RD (2012) Naltrindole inhibits human multiple myeloma cell proliferation in vitro and in a murine xenograft model in vivo. J Pharmacol Exp Ther 342, 273-287

27. Louis KS and Siegel AC (2011) Cell viability analysis using trypan blue: manual and automated methods. Methods Mol Biol 740, 7-12

28. Qian AR, Gao X, Zhang W et al (2013) Large Gradient High Magnetic Fields Affect Osteoblast Ultrastructure and Function by Disrupting Collagen I or Fibronectin/alphabeta1 Integrin. PLoS One 8, e51036 